\title{
Morphology and Phylogeny of a New Species of Anaerobic Ciliate, Trimyema finlayi n. sp., with Endosymbiotic Methanogens
}

\author{
William H. Lewis ${ }^{1,2 *}$, Kacper M. Sendra ${ }^{1}$, T. Martin Embley ${ }^{1}$ and Genoveva F. Esteban ${ }^{2 *}$ \\ ${ }^{1}$ Institute for Cell and Molecular Biosciences, Newcastle University, Newcastle upon Tyne, United Kingdom, ${ }^{2}$ Bournemouth \\ University, Faculty of Science and Technology, Department of Life \& Environmental Sciences, Poole, United Kingdom
}

OPEN ACCESS

Edited by:

Suhelen Egan,

University of New South Wales,

Australia

Reviewed by:

Roxanne Beinart,

University of Rhode Island,

United States

Maher Gtari,

Carthage University, Tunisia

*Correspondence:

William H. Lewis

william.lewis@icm.uu.se

Genoveva F. Esteban

gesteban@bournemouth.ac.uk

Specialty section:

This article was submitted to

Microbial Symbioses,

a section of the journal

Frontiers in Microbiology

Received: 30 October 2017 Accepted: 22 January 2018

Published: 19 February 2018

Citation:

Lewis WH, Sendra KM, Embley TM and Esteban GF (2018) Morphology and Phylogeny of a New Species of Anaerobic Ciliate, Trimyema finlayi

n. sp., with Endosymbiotic Methanogens.

Front. Microbiol. 9:140

doi: 10.3389/fmicb.2018.00140
Many anaerobic ciliated protozoa contain organelles of mitochondrial ancestry called hydrogenosomes. These organelles generate molecular hydrogen that is consumed by methanogenic Archaea, living in endosymbiosis within many of these ciliates. Here we describe a new species of anaerobic ciliate, Trimyema finlayi n. sp., by using silver impregnation and microscopy to conduct a detailed morphometric analysis. Comparisons with previously published morphological data for this species, as well as the closely related species, Trimyema compressum, demonstrated that despite them being similar, both the mean cell size and the mean number of somatic kineties are lower for $T$. finlayi than for $T$. compressum, which suggests that they are distinct species. This was also supported by analysis of the 18S rRNA genes from these ciliates, the sequences of which are $97.5 \%$ identical (6 substitutions, 1479 compared bases), and in phylogenetic analyses these sequences grouped with other $18 \mathrm{~S}$ rRNA genes sequenced from previous isolates of the same respective species. Together these data provide strong evidence that $T$. finlayi is a novel species of Trimyema, within the class Plagiopylea. Various microscopic techniques demonstrated that $T$. finlayi n. sp. contains polymorphic endosymbiotic methanogens, and analysis of the endosymbionts' 16S rRNA gene showed that they belong to the genus Methanocorpusculum, which was confirmed using fluorescence in situ hybridization with specific probes. Despite the degree of similarity and close relationship between these ciliates, T. compressum contains endosymbiotic methanogens from a different genus, Methanobrevibacter. In phylogenetic analyses of $16 \mathrm{~S}$ rRNA genes, the Methanocorpusculum endosymbiont of $T$. finlayi $\mathrm{n}$. sp. grouped with sequences from Methanomicrobia, including the endosymbiont of an earlier isolate of the same species, 'Trimyema sp.,' which was sampled approximately 22 years earlier, at a distant $(\sim 400 \mathrm{~km})$ geographical location. Identification of the same endosymbiont species in the two separate isolates of $T$. finlayi n. sp. provides evidence for spatial and temporal stability of the MethanocorpusculumT. finlayi n. sp. endosymbiosis. T. finlayi $\mathrm{n}$. sp. and T. compressum provide an example of two closely related anaerobic ciliates that have endosymbionts from different methanogen genera, suggesting that the endosymbionts have not co-speciated with their hosts.

Keywords: anaerobic, ciliate, endosymbiont, methanogen, Trimyema, phylogeny, Methanocorpusculum 


\section{INTRODUCTION}

Known species of the genus Trimyema (class: Plagiopylea, phylum: Ciliophora) are all anaerobic and inhabit diverse environments including freshwater, marine and hypersaline sediments, sewage tanks and hydrothermal vents (Baumgartner et al., 2002; Esteban and Finlay, 2004; Shinzato et al., 2007; Cho et al., 2008). During adaptation to their anaerobic lifestyle, the mitochondria of these ciliates have evolved into hydrogenosomes, mitochondrial homologs that produce $\mathrm{H}_{2}$, which is consumed by endosymbiotic methanogenic Archaea (phylum: Euryarchaeota) that live inside the ciliate cells (Augustin et al., 1987; Wagener and Pfennig, 1987; Zwart et al., 1988; Finlay et al., 1993; Lynn, 2008).

Like Trimyema, some other microbial eukaryotes can have methanogenic endosymbionts, but they are particularly common in anaerobic ciliates (van Bruggen et al., 1983, 1985; Broers et al., 1990; Finlay et al., 1994; Fenchel and Finlay, 1995). Except for in a handful of cases (Embley et al., 1992a,b; Finlay et al., 1993; Shinzato et al., 2007), the identity of the endosymbiont species has not been reliably established using such methods as speciesspecific in situ probing. Phylogenetic analyses have provided evidence that methanogenic endosymbionts of some ciliates do not evolve in parallel with their hosts and in some cases have been replaced by a new methanogen species (Finlay et al., 1993; van Hoek et al., 2000a). This indicates that the association between methanogenic endosymbionts and their hosts is not entirely stable, and it is possible that a single host species could contain different endosymbionts in specific habitats and at specific times (Embley and Finlay, 1994).

Balanced against the idea that methanogenic endosymbionts are not retained over longer evolutionary time periods, there is evidence from some anaerobic ciliates that their methanogenic endosymbionts are transmitted vertically, and therefore are retained over the evolutionary short-term. For example, the endosymbionts of the ciliate Plagiopyla frontata divide in synchrony with their host, which ensures that each daughter host cell receives a number of endosymbionts similar to the number that the mother cell had before division (Fenchel and Finlay, 1991). Likewise, the methanogenic endosymbionts in the ciliate Metopus palaeformis were shown to divide at a rate that would ensure a stable population size from one generation of the host to the next (Finlay and Fenchel, 1992). These examples suggest that at least in some anaerobic ciliates, methanogenic endosymbionts have adapted to being vertically transmitted and are not continually replaced by a new methanogen species between host generations. Resampling of endosymbionts from the same host species, isolated at different times and locations, would provide a test of these ideas, and would help us to understand the extent to which these endosymbionts have been retained during the evolutionary history of their hosts.

In 1993, Finlay and colleagues isolated a species of Trimyema that was referred to as 'Trimyema sp.' in several subsequent publications (Embley and Finlay, 1993, 1994; Embley et al., 1995, 2003; Fenchel and Finlay, 1995; Embley, 2006). 'Trimyema sp.' was described as sharing some morphological similarities to the species Trimyema compressum but some distinctions were also highlighted: 'Trimyema sp.' had fewer somatic kineties than $T$. compressum and both species differed in the structure of their brosse and in their oral infraciliature (Finlay et al., 1993). In the present study, 'Trimyema sp.' was re-isolated and cultured, identified based on morphometric and molecular data, and demonstrated to be closely related to, but distinct from, T. compressum. This new isolate represents a new taxonomic species, which here we describe as Trimyema finlayi $\mathrm{n}$. sp. The species of endosymbiotic methanogen in T. finlayi was identified by sequencing its $16 \mathrm{~S}$ rRNA gene, and validated using fluorescent in situ hybridization (FISH). A phylogenetic approach was used to investigate the relationship of $T$. finlayi to other ciliates, as well as the relationship of its endosymbiotic methanogen to the methanogenic endosymbiont of T. compressum and to other methanogenic Archaea. Comparison of the endosymbiont 16S rRNA gene sequences isolated from two closely-related species of ciliates ( $T$. finlayi n. sp. and T. compressum), as well as those from two isolates of the same species (T. finlayi n. sp. and 'Trimyema sp.') sampled 22 years, and over $400 \mathrm{~km}$ apart, provide new insights into spatial and temporal stability of endosymbiosis between anaerobic ciliates and methanogenic Archaea.

\section{MATERIALS AND METHODS}

\section{Isolation and Culture of Organisms}

Sediment was collected from a freshwater pond located at the East Stoke Fen Nature Reserve $(50.679159,-2.191654)$, close to Wareham, Dorset (United Kingdom), on the floodplain of the river Frome. These samples were collected in April 2013, at which time the depth of the pond did not exceed $1 \mathrm{~m}$. The collected sediment samples were transferred to glass hypo-vials, to which Soil Extract with added Salts (SES) medium was added, prepared according to instructions available from Culture Collection of Algae and Protozoa (CCAP) ${ }^{1}$. Approximately $5 \mathrm{mg}$ of crushed dried cereal leaves and one wheat grain were added to each culture to encourage growth of the naturally existing prokaryotic flora, providing food for the ciliates. The hypo-vials were sealed and their headspace flushed with nitrogen gas for 3 min to remove oxygen, maintaining anoxic conditions within the vials. These enrichment cultures were left to grow for 2 weeks until species of anaerobic ciliates could be microscopically observed in aliquots removed from the cultures. Mono-ciliate cultures were obtained by transferring individual cells to hypo-vials of fresh anoxic culture medium using a glass micropipette. Subculturing was performed monthly by dividing the cultures and then adding fresh media, cereal leaves and wheat grains. All cultures were continually incubated at $18^{\circ} \mathrm{C}$.

\section{DIC Microscopy of Ciliate Cells and F420-Autofluorescence Imaging of Methanogenic Endosymbionts}

Living or fixed (4\% paraformaldehyde) ciliate cells were imaged using an Olympus BH-2 light microscope and photographed with a Micropublisher 3.3 RTV mounted camera (QImaging).

\footnotetext{
${ }^{1}$ https://www.ccap.ac.uk/media/documents/SES.pdf
} 

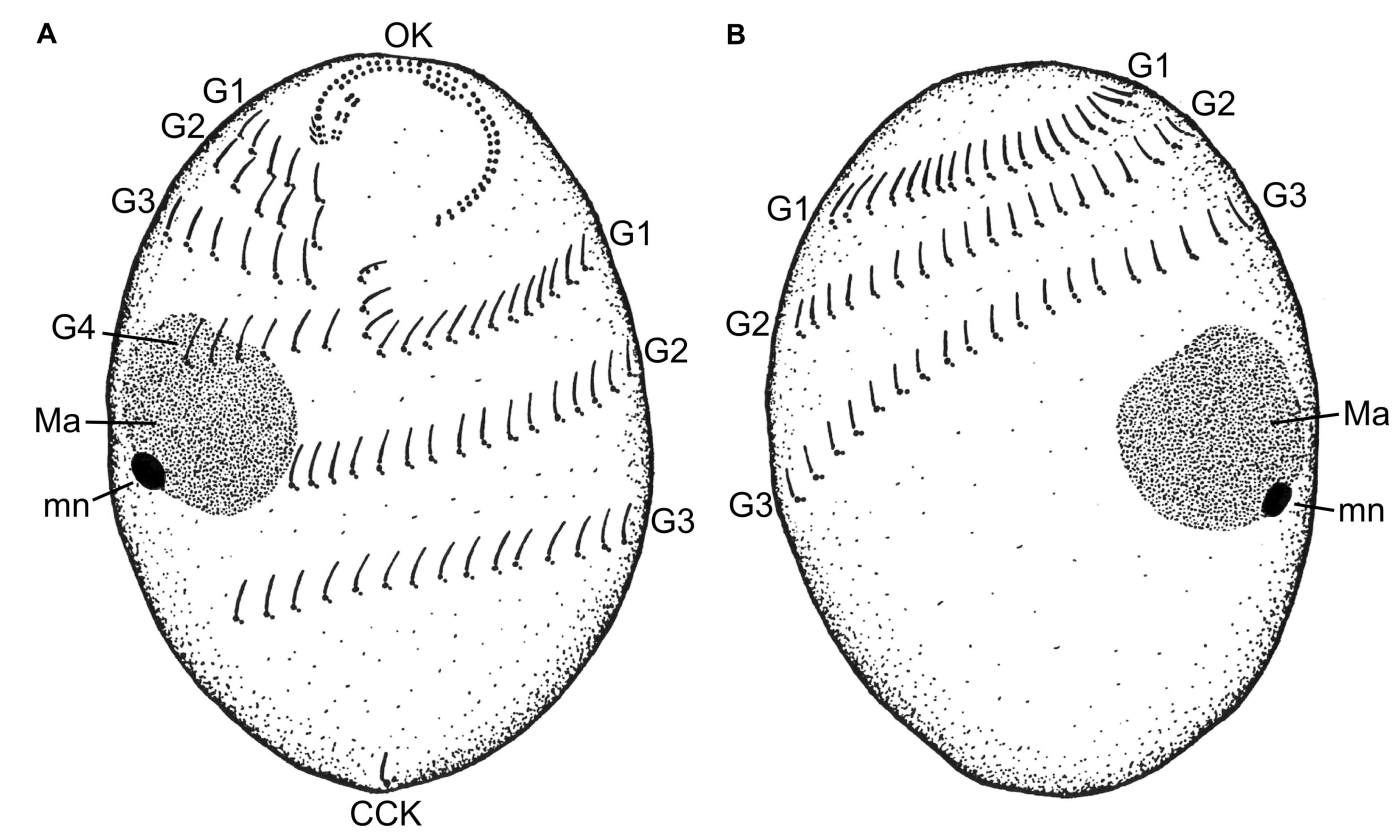

FIGURE 1 | A schematic drawing of the (A) ventral and (B) dorsal sides of a Trimyema finlayi $\mathrm{n}$. sp. cell. CCK, caudal cilium kinety; G1, first ciliary girdle; G2, second ciliary girdle; G3, third ciliary girdle; G4, fourth ciliary girdle; Ma, macronucleus; mn, micronucleus; OK, oral kineties.

Cell measurements were taken from the images using QCapture Pro software (QImaging). The same microscope and camera was used to detect F420 auto-fluorescence emitted by endosymbiotic methanogens whilst illuminated with UV light (Doddema and Vogels, 1978). To be imaged using this method cells of ciliates were fixed in $4 \%$ paraformaldehyde and transferred to a Isopore ${ }^{\mathrm{TM}}$ polycarbonate membrane filter (Merck-Millipore), mounted between a microscope slide and cover slip using FF immersion oil (Cargille). Silver carbonate staining of cells was performed as described by Fernández-Galiano (1994).

\section{DNA Amplification and Sequencing}

Polymerase chain reaction was used to amplify the $18 \mathrm{~S}$ rRNA gene from ciliate cells using KOD Hot Start DNA Polymerase (Merck-Millipore) with the manufacturer's standard protocol. Five cells were isolated by micropipette, washed three times in sterile PBS, and then transferred to an unsealed microcentrifuge tube, which was then dried at $80^{\circ} \mathrm{C}$ for 30 min inside a tissue culture hood. This provided the DNA template for the PCR reaction, to which $50 \mu \mathrm{l}$ of PCR reaction mixture was added. Forward (5'-AYCTGGTTGATYYTGCCAG) and reverse (5'-TGATCCATCTGCAGGTTCACCT) primers (Embley et al., 1992b) were used in an initial PCR reaction to amplify an 1767 base pair fragment of the eukaryotic $18 \mathrm{~S}$ rRNA gene. The product of this reaction was purified using a QIAquick PCR Purification Kit (QIAGEN) and used as the DNA template of secondary, semi-nested, PCR reactions. One of the semi-nested reactions used the forward primer from the first reaction with the reverse primer EK-1269R (5'-AAGAACGGCCATGCACCAC) (LópezGarcía et al., 2001), and the other semi-nested reaction used the forward primer EK-555F (5'-AGTCTGGTGCCAGCAGCCGC)
(López-García et al., 2001) and the reverse primer from the first reaction. The same PCR methods were used to amplify the $16 \mathrm{~S}$ rRNA gene of the T. finlayi endosymbiotic methanogen, except the forward primer 340F (5'-CCCTAYGGGGYGCASCAG) (Gantner et al., 2011) and the reverse primer $1100 \mathrm{~A}$ (5'-TGGGTCTCGCTCGTTG) (Embley et al., 1992b) were used, without a secondary semi-nested reaction.

Thermal cycling conditions used in all PCR reactions were the same as those described by Embley et al. (1992b), except with the addition of an initial heating step at $95^{\circ} \mathrm{C}$ for $2 \mathrm{~min}$, which was required for the activation of the KOD polymerase. The products of these two semi-nested reactions were purified from a 1\% agarose gel using a QIAquick Gel Extraction Kit (QIAGEN), ligated into pJET 1.2 plasmids and cloned using a CloneJET PCR Cloning Kit (Life Technologies) in DH5 $\alpha$ cells. Plasmids were purified from overnight cultures using a QIAprep Spin Miniprep Kit (QIAGEN) and five clones for each PCR product were Sanger sequenced in both directions by GATC Biotech using plasmid-specific sequencing primers provided in the cloning kit. Sequencing reads were trimmed and assembled into a complete sequence using the program Sequencher 5.4.6 (Gene Codes Corporation).

\section{Sequence and Phylogenetic Analysis}

For ciliate $18 \mathrm{~S}$ and methanogen $16 \mathrm{~S}$ rRNA gene phylogenies, sequences obtained in the present study were aligned with sequences downloaded from GenBank, using the program MUSCLE 3.8.31 (Edgar, 2004). Conserved sites within each dataset were selected and concatenated with the program Gblocks 0.91b (Castresana, 2000). The program jModelTest 2.1.10 (Darriba et al., 2012) selected GTR $+\Gamma+\mathrm{I}$ as the best-fitting 
model for both alignment datasets. Maximum likelihood analysis was performed with the program RAxML 8.2.4 (Stamatakis, 2014) and statistical support for internal nodes was assessed with 1000 bootstrap replicates. Bayesian analysis was performed using the program MrBayes 3.2.2 (Ronquist and Huelsenbeck, 2003). Two sets of four MCMC chains ran for 1,000,000 generations and were sampled every 100 generations, after which $25 \%$ of samples were discarded as burn-in and the standard deviation of split frequencies was below 0.01 .

\section{Fluorescence in Situ Hybridization (FISH)}

The endosymbiotic methanogen of $T$. finlayi was identified by FISH using the Methanocorpusculum oligonucleotide probe, SYM5 (5'-CTGCATCGACAGGCACT) (Finlay et al., 1993), dual labeled with 6-Fam and the positivecontrol Archaea-specific oligonucleotide probe, ARCH915 (5'-GTGCTCCCCCGCCAATTCCT) (Stahl and Amann, 1991), dual-labeled with Cy3. Both probes were synthesized by biomers.net. Cells were isolated from culture using a micropipette, fixed in $4 \%$ paraformaldehyde at $4^{\circ} \mathrm{C}$ and transferred to poly-L-lysine coated slides. Sample dehydration, probe hybridization and washing were the same as described in Daims et al. (2005). Dried, hybridized samples were mounted on glass cover slides using ProLong Diamond antifade mountant. Z-sections were imaged using a confocal microscope (A1R, Nikon) with a $63 \mathrm{x} / 1.4$ objective lens. Vertical z-stacks were deconvolved using Huygens deconvolution software (Scientific Volume Imaging B.V.) with empirically measured point spread functions extracted from images of $0.1 \mu \mathrm{m}$ TetraSpeck $^{\text {TM }}$ Microspheres (Thermo Fisher). Maximum intensity Z-projection images were reconstructed using Fiji (Schindelin et al., 2012).

\section{Transmission Electron Microscopy}

Samples were prepared for transmission electron microscopy (TEM) by centrifuging $200 \mathrm{ml}$ of ciliate cultures at $1500 \times g$ for $45 \mathrm{~min}$. Supernatant was then carefully removed to leave the pellets intact, which were transferred to microcentrifuge tubes. Cells were fixed in 2.5\% glutaraldehyde in 0.15 M HEPESbuffer at $4^{\circ} \mathrm{C}$. The remaining sample preparation, including post-fixation and embedding, and also imaging of the samples, was performed by Benoît Zuber and Beat Haenni, Microscopy Imaging Center (MIC), Institute of Anatomy, University of Bern, Switzerland, using methods that have been described previously (Tschanz et al., 2003).

\section{RESULTS}

\section{Morphology of Trimyema finlayi n. sp.}

The cell shape of $T$. finlayi is a fusiform ellipsoid, tapering toward both the anterior and posterior ends (Figure 1). The cell body measured in vivo from 60 cells had a length of 27.7-39.9 $\mu \mathrm{m}$ and a width of 17.6-26.6 $\mu \mathrm{m}$ (Table 1). T. finlayi cells have a single macronucleus, which is positioned off-center from the vertical axis and toward the anterior end of the cell (Figures 2A-E,L). The macronucleus is strongly stained by the silver carbonate method (Figures 2A-F) and is therefore easy to visualize. The small micronucleus was observed in close proximity to the macronucleus (Figure 1) and could not be easily resolved in most of the images from stained specimens. Each cell had 34-45 somatic kineties, organized in longitudinal rows that create the appearance of four ciliary girdles, spiraling obliquely around the cell surface toward the posterior end (Table $\mathbf{1}$ and Figures $\mathbf{2 A - D , J , K ) . ~}$ The cell has a single caudal cilium (Figures 2C,D), close to which is positioned the cytoproct in the most posterior third of the cell surface (Figures 2D,F). The oral cavity is located close to the n-kinety (Figures 2B,C). The endosymbiotic methanogens within the ciliate cell appear to form clusters with hydrogenosomes and are distributed throughout the cytoplasm (Figures 2G-I, 3).

TABLE 1 | Morphometric data characterizing Trimyema finlayi.

\begin{tabular}{|c|c|c|c|c|c|c|c|c|c|}
\hline Characteristics & Method & $\bar{x}$ & $M$ & $S D$ & $S E$ & CV & Min & Max & $n$ \\
\hline Body length ( $\mu \mathrm{m})$ & IV & 34.2 & 34.0 & 2.9 & 0.4 & 0.1 & 27.7 & 39.9 & 60 \\
\hline Body length ( $\mu \mathrm{m})$ & $\mathrm{FF}$ & 35.2 & 35.2 & 3.6 & 0.6 & 0.1 & 29.3 & 43.4 & 37 \\
\hline Body width ( $\mu \mathrm{m})$ & IV & 22.1 & 22.1 & 2.1 & 0.3 & 0.1 & 17.6 & 26.6 & 60 \\
\hline Body width ( $\mu \mathrm{m})$ & $\mathrm{FF}$ & 25.5 & 25.4 & 3.6 & 0.6 & 0.1 & 19.1 & 34.6 & 37 \\
\hline Macronuclei number & SC & 1.0 & 1.0 & 0.0 & 0.0 & 0.0 & 1.0 & 1.0 & 15 \\
\hline Oral ciliary rows, number & SC & 3.0 & 3.0 & 0.0 & 0.0 & 0.0 & 3.0 & 3.0 & 15 \\
\hline Kinetids in oral ciliary row & SC & 44.3 & 45.0 & 2.1 & 1.2 & 0.0 & 42.0 & 46.0 & 3 \\
\hline Ciliary girdles on cell body & SC & 4.0 & 4.0 & 0.0 & 0.0 & 0.0 & 4.0 & 4.0 & 15 \\
\hline First ciliary girdle, number of kinetids & SC & 39.3 & 39.0 & 2.7 & 0.7 & 0.1 & 34.0 & 43.0 & 15 \\
\hline Second ciliary girdle, number of kinetids & SC & 42.6 & 43.0 & 2.2 & 0.6 & 0.1 & 39.0 & 45.0 & 15 \\
\hline Third ciliary girdle, number of kinetids & SC & 41.3 & 41.0 & 1.1 & 0.3 & 0.0 & 40.0 & 43.0 & 15 \\
\hline Fourth ciliary girdle, number of kinetids & SC & 5.7 & 6.0 & 0.5 & 0.1 & 0.1 & 5.0 & 6.0 & 15 \\
\hline Number of $\mathrm{N}$ kinetids & SC & 3.2 & 3.0 & 0.4 & 0.1 & 0.1 & 3.0 & 4.0 & 15 \\
\hline Caudal cilia number & $\mathrm{FF}$ & 1.0 & 1.0 & 0.0 & 0.0 & 0.0 & 1.0 & 1.0 & 37 \\
\hline
\end{tabular}

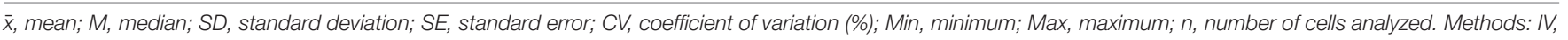
in vivo; FF, fixed $4 \%$ formalin; SC, silver carbonate staining. 
A
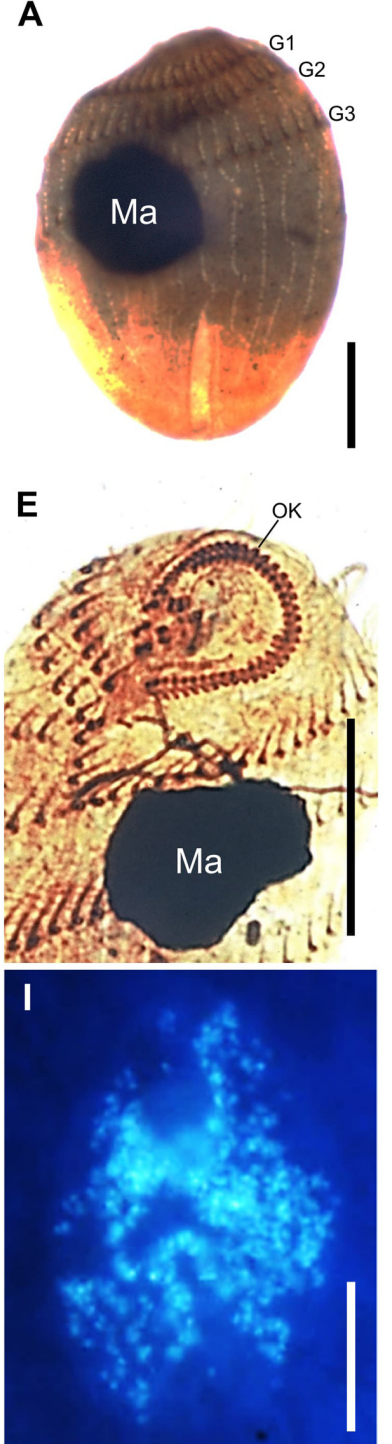

\section{B}

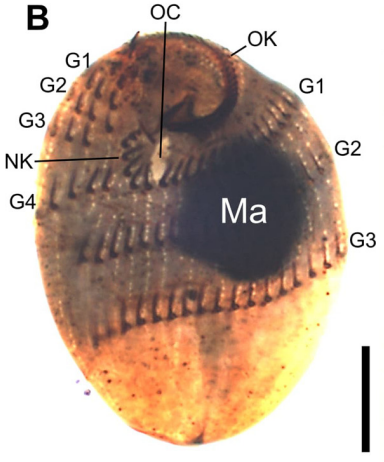

$\mathbf{F}$
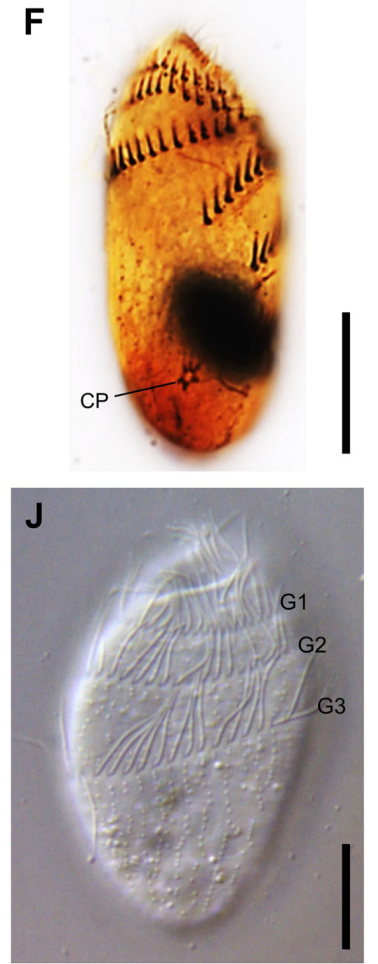

C
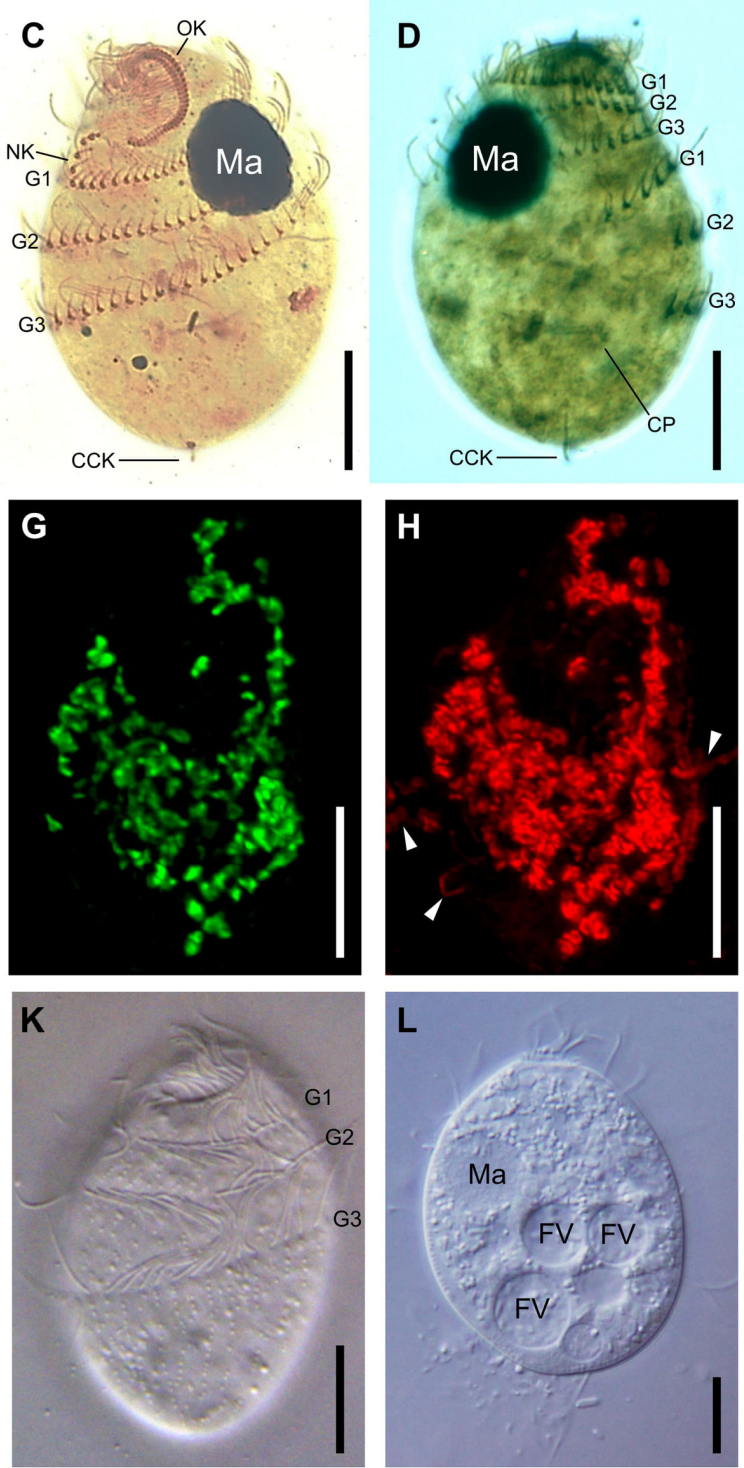

FIGURE 2 | Microscopic imaging of T. finlayi n. sp. whole cells. DIC images of silver carbonate impregnated cells, (A,B) and (C,D) show two sides of the same cells, (E) squashed cell showing oral kineties, (F) squashed cell showing cytoproct. (G,H) Maximum intensity projection of a Z-stack of confocal images across a single T. finlayi cell double-labeled with two FISH probes. (G) Methanocorpusculum-specific probe (SYM5) dual-labeled with 6-FAM. (H) Archaea-specific probe (ARCH915) dual-labeled with Cy3, white arrows indicate extracellular Archaea that were not labeled by the probe SYM5 (G). (I) F420 auto-fluorescence. (J-L) In vivo DIC images. CCK, caudal cilium kinety; CP, cytoproct; FV, food vacuole; G1, first ciliary girdle; G2, second ciliary girdle; G3, third ciliary girdle; G4, fourth ciliary girdle; MA, macronucleus; NK, N-kineties; OC, oral cavity; OK, oral kineties. Scale bars $=10 \mu \mathrm{m}$.

Holotype and paratypes: A permanent preparation with silver-impregnated specimens has been deposited in the Natural History Museum, London (United Kingdom) (accession: NHMUK 2018.1.30.1) and the species is registered with ZooBank (lsid:zoobank.org:pub:12910A6B-D834-43148812-0039F87D6DD2).

Type locality: East Stoke Fen Nature Reserve, East Stoke, Wareham, Dorset (United Kingdom) (50.679159, $-2.191654)$.

Etymology: finlayi, dedicated to Professor Bland J. Finlay, in recognition of his many contributions to understanding the ecology of anaerobic ciliates and their endosymbionts, and his impact on the field of microbial ecology more generally.

\section{Phylogenetic Relationships of Trimyema finlayi $\mathrm{n}$. sp.}

Phylogenetic analysis of the 18S rRNA gene sequence for T. finlayi (accession number: MF074215) (Figure 4) suggests that it is most closely related to 'Trimyema sp.' (bootstrap support $=100$, posterior probability $=1$ ) and comparable sequenced regions of their $18 \mathrm{~S}$ rRNA genes are $99.6 \%$ identical 


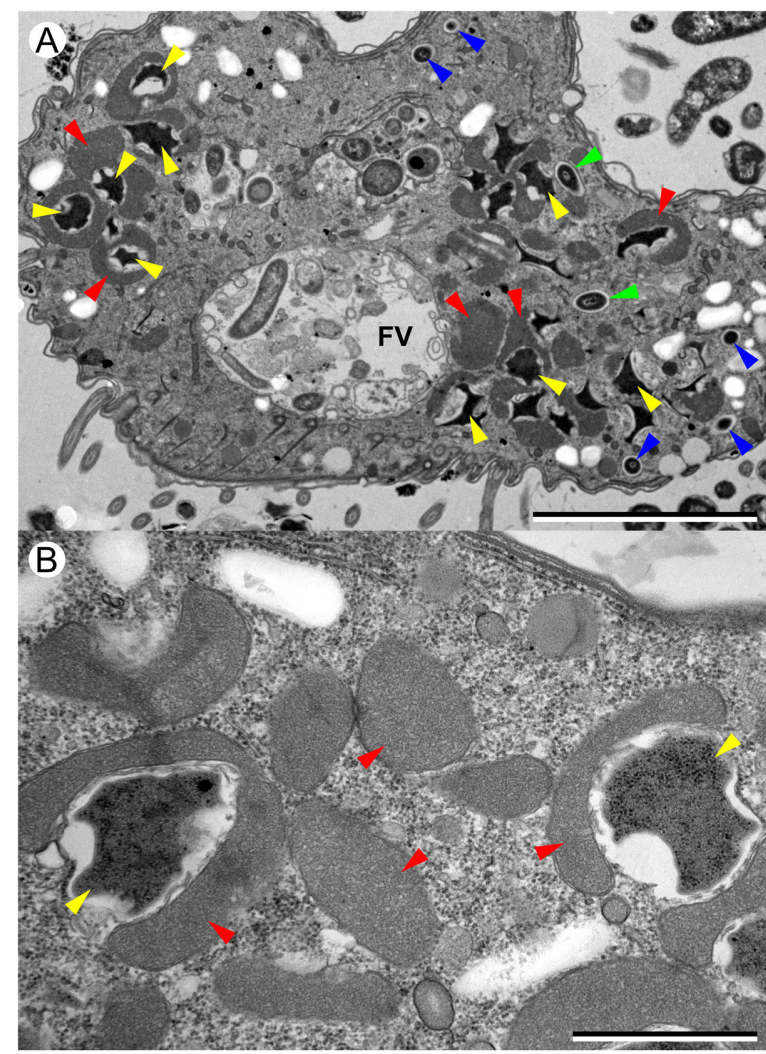

FIGURE 3 | Transmission electron microscopy (TEM) images of T. finlayi $\mathrm{n}$. sp. showing polymorphic methanogenic endosymbionts and hydrogenosomes (red arrowheads). Disc-shaped (blue arrowheads) and stellate form (yellow arrowheads) morphotypes are shown, as well as intermediate stages (green arrowheads). FV, food vacuole. Scale bars $(\mathbf{A})=5 \mu \mathrm{m}, \mathbf{( B )}=1 \mu \mathrm{m}$.

when aligned. These two sequences form a sister group (bootstrap support $=94$, posterior probability $=0.96)$ to a clade containing two sequences from $T$. compressum (bootstrap support $=100$, posterior probability $=1$ ) and they are also more closely related to other Trimyema sequences than other species of Plagiopylea (Figure 4).

\section{Identification and Morphology of Endosymbiotic Methanogens Living in Trimyema finlayi $\mathrm{n} . \mathrm{sp}$.}

F420 autofluorescence (Figure 2I) indicated the presence of methanogens within cells of $T$. finlayi. In order to identify the species of these methanogens, a 16S rRNA gene was sequenced from isolated ciliate cells, which was $99 \%$ identical to sequences from several species of the genus Methanocorpusculum in GenBank, including Methanocorpusculum parvum and Methanocorpusculum aggregans. In FISH experiments, Archaea labeled with a Methanocorpusculum-specific oligonucleotide probe, SYM5, were localized inside $T$. finlayi cells, but not outside (Figure 2G). A positive-control Archaea-specific oligonucleotide probe, ARCH915, bound to the endosymbiotic methanogens, as well as extracellular Archaea present in the sample (Figure $\mathbf{2 H}$ ).

Transmission electron microscopy images indicate that the endosymbiotic methanogens in $T$. finlayi are polymorphic, consisting of two main morphotypes: cells of the first morphotype appear smaller and more round (Figure 3, blue arrowheads) and are have previously been described as 'disc-shaped' (Finlay et al., 1993). Cells of the second morphotype are larger and more irregular in shape (Figure 3, yellow arrowheads), with their cell walls more invaginated; cells of this morphotype have previously been described as 'stellate forms' (Finlay et al., 1993). The endosymbiont cells of the stellate-form morphotype are also typically closely associated with hydrogenosomes (Figure 3, red arrowheads) and in some cases appear almost completely encapsulated by them. In addition, there appear to be some intermediate forms between these two morphotypes (Figure 3, green arrowheads), suggesting that the endosymbionts undergo transformation from one form to the other, as observed by Finlay et al. (1993).

Several findings support the idea that the endosymbionts are two morphotypes of the same species (Finlay et al., 1993): firstly, based on TEM images (Figure 3), in the case of all morphotypes, the center of the methanogen is electron-dense and is surrounded by a less electron-dense outline that varies in thickness. Secondly, the endosymbionts appear similar when labeled with different FISH-probes (Figures 2G,H), as well as when imaged based on their F420-autofluorsence (Figure 2I). Additionally, each of these images looks like those of earlier isolates (i.e., 'Trimyema sp.'), which were made using similar methods (Finlay et al., 1993). Finally, the Archaea-specific FISH-probe (Figure 2H) co-localizes with the Methanocorpusculum-specific FISH-probe (Figure 2G), suggesting that all of the archaeal cells within T. finlayi are the same species of the genus Methanocorpusculum.

\section{Phylogenetic Relationships of Endosymbiotic Methanogens from Trimyema Species and Their Free-Living Relatives}

In order to investigate the relationship between the endosymbiotic methanogens of Trimyema species and other methanogenic Archaea, the 16S rRNA gene of the endosymbionts from T. finlayi (accession number: MF074216) was sequenced (from hand-picked and washed ciliate cells) and analyzed phylogenetically, together with the 16S rRNA genes of other methanogens (Figure 5). The endosymbiotic methanogens of T. finlayi and 'Trimyema sp.' (Finlay et al., 1993) grouped together (bootstrap support $=100$, posterior probability $=0.98$ ), and they both formed a clade with the free-living methanogen species Methanocorpusculum labreanum (bootstrap support $=100$, posterior probability $=1$ ), within a larger clade that includes sequences from other species in the order Methanomicrobiales (bootstrap support $=100$, posterior probability $=1$ ). Identification of only a single $16 \mathrm{~S}$ rRNA gene sequence from T. finlayi (this study) and 'Trimyema sp.' (Finlay et al., 1993) isolates, provides further support for the hypothesis that the two types of archaeal cells, observed 


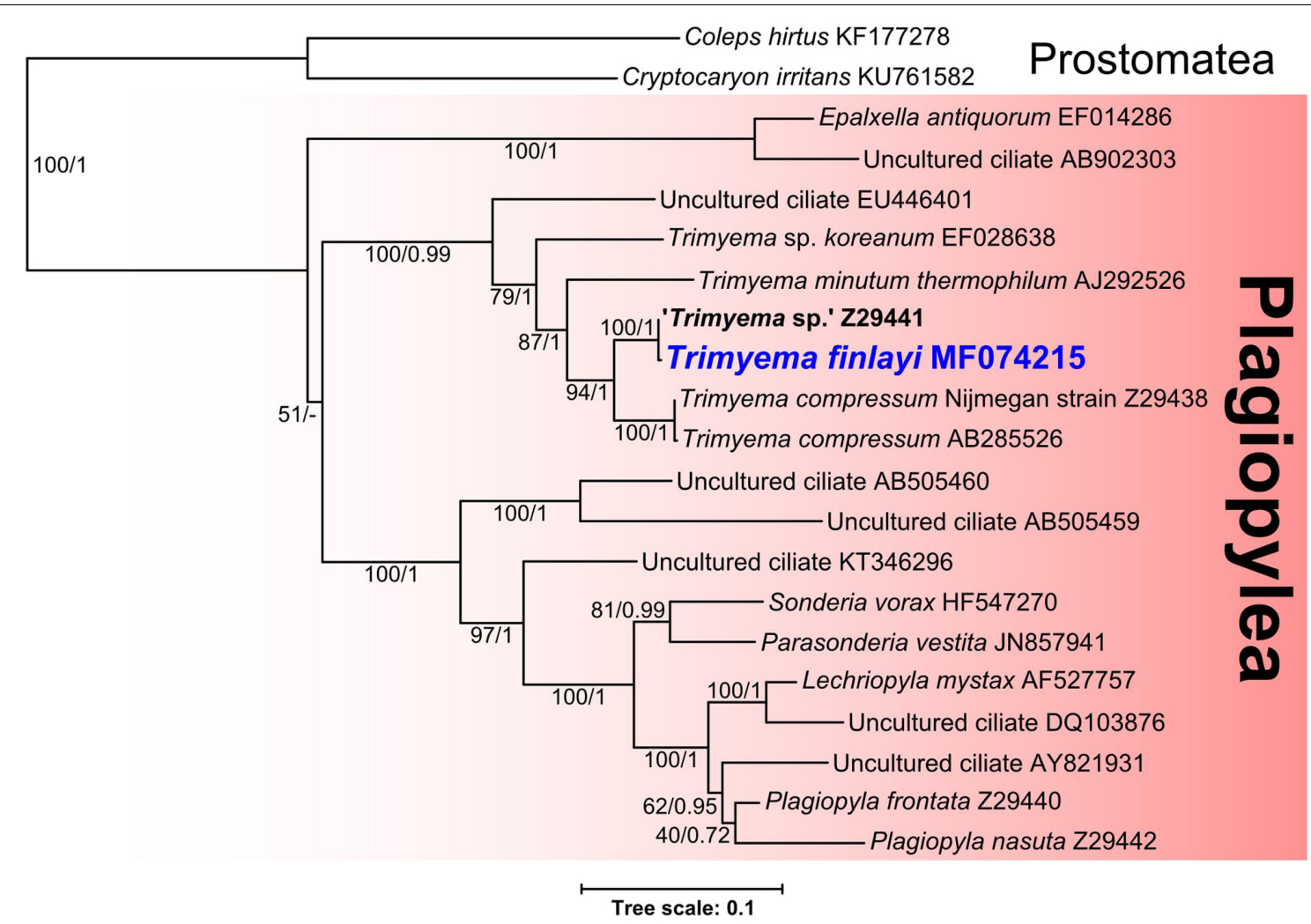

FIGURE 4 | Bayesian phylogeny inferred from 1640 nucleotide alignment of $18 \mathrm{~S}$ rRNA genes of Plagiopylea species using the GTR $+\Gamma+$ I model. Support values represent maximum likelihood bootstrap support/Bayesian posterior probabilities. Scale bar represents the number of substitutions per site.

inside the T. finlayi cytosol in TEM images (Figure 3), are two morphotypes of a single archaeal species. The endosymbiotic methanogen of the ciliate $T$. compressum did not group with the endosymbionts of ciliates from the same genus, 'Trimyema sp.' and T. finlayi, as was suggested previously (Shinzato and Kamagata, 2010), and is consistent with the hypothesis that the endosymbiosis has been established more than once during the evolution of the Trimyema lineage. Instead the endosymbiont of $T$. compressum forms a clade with species in the order Methanobacteriales (bootstrap support $=100$, posterior probability $=1$ ) and is most closely related to the free-living methanogen Methanobrevibacter arboriphilus (bootstrap support $=100$, posterior probability $=1$ ). This is consistent with a previous study that identified the methanogenic endosymbiont of $T$. compressum as a member of the Methanobacteria genus Methanobrevibacter by using FISH with a species-specific probe (Shinzato et al., 2007).

\section{DISCUSSION}

Morphological descriptions of $T$. compressum differ between publications (Augustin et al., 1987; Wagener and Pfennig, 1987; Serrano et al., 1988) and a general consensus seems to be lacking. Therefore morphological parameters of $T$. finlayi were compared to three previously published descriptions of
T. compressum (Augustin et al., 1987; Wagener and Pfennig, 1987; Serrano et al., 1988) as well as a previously published partial description of 'Trimyema sp.' (Finlay et al., 1993) (Table 2). The measured mean length of $T$. finlayi $(34.7 \mu \mathrm{m})$ was lower than the mean length of T. compressum (39.05-65.9 $\mu \mathrm{m})$, based on all three descriptions and falls within the range that was specified for 'Trimyema sp.' (30-50 $\mu \mathrm{m})$. The range in number of somatic kineties recorded for 'Trimyema sp.' (37-40) falls within the range measured for $T$. finlayi (34-45), whereas the range in number of somatic kineties for $T$. compressum is systematically higher (50-60) (Augustin et al., 1987; Serrano et al., 1988).

Phylogenetic analysis of the $18 \mathrm{~S}$ rRNA genes sequenced from these ciliates (Figure 4) suggests that T. finlayi and 'Trimyema sp.' form a clade (bootstrap support $=100$, posterior probability $=1$ ) that is a sister group to sequences from T. compressum (bootstrap support $=94$, posterior probability $=1$ ). The small number of nucleotide differences (6 substitutions; 1479 compared bases) between the $18 \mathrm{~S}$ rRNA gene sequences from $T$. finlayi and 'Trimyema sp.' could be a consequence of inter-strain differences, due to them being isolated at different times and locations (South and North of England, respectively). Alternatively, since the 'Trimyema sp.' sequence (accession number: Z29441.1) contains 12 ambiguous bases, this suggests that the overall quality of the sequence is relatively low, and therefore these differences between the two sequences could be the result of sequencing 


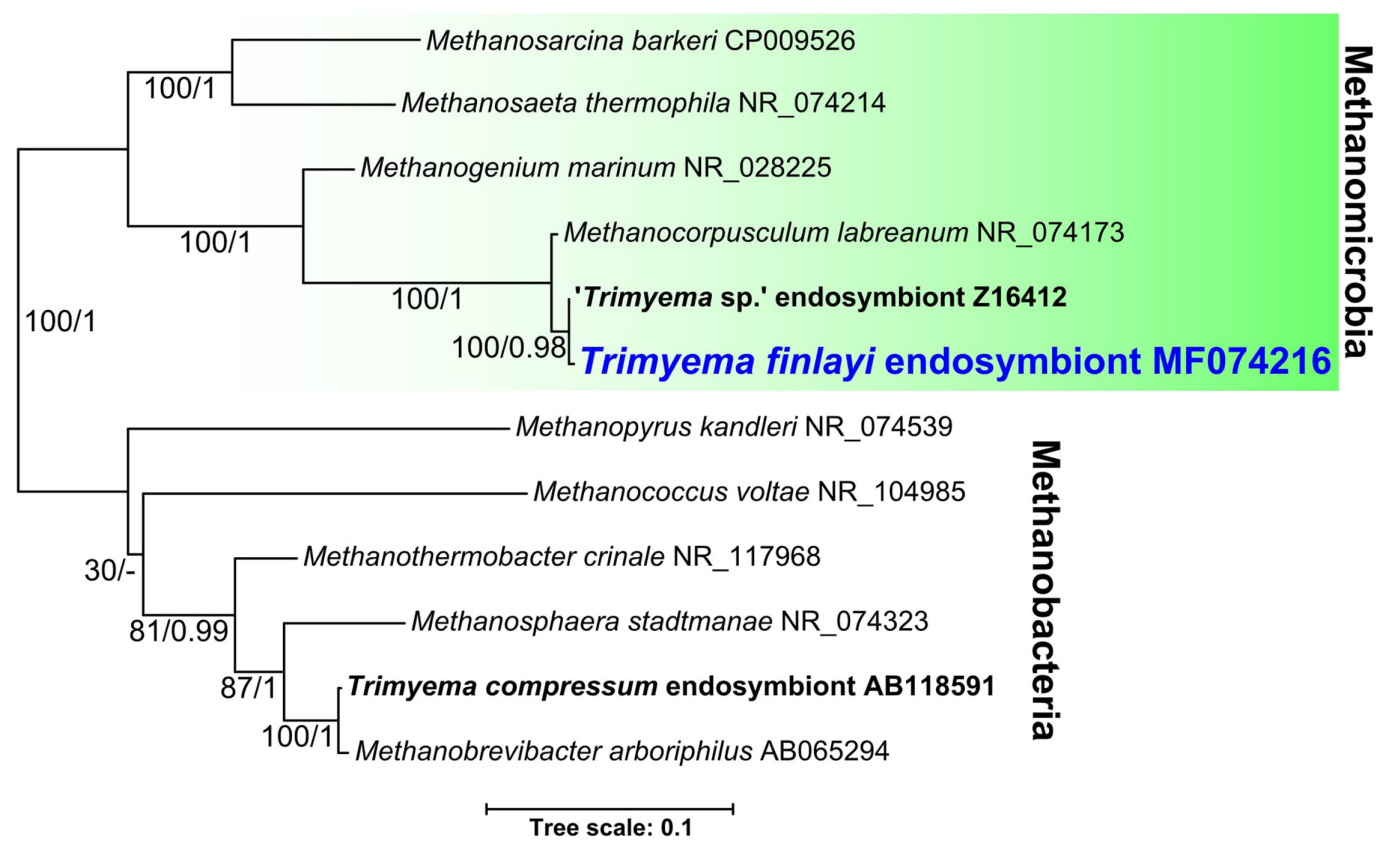

FIGURE 5 | Bayesian phylogeny inferred from a 1372 nucleotide alignment of methanogenic Archaea 16S rRNA genes using the GTR $+\Gamma+$ I model. Support values represent maximum likelihood bootstrap support/Bayesian posterior probabilities. Scale bar represents the number of substitutions per site.

TABLE 2 | Comparison of morphometric data collected in separate studies for species of Trimyema.

\begin{tabular}{|c|c|c|c|c|c|}
\hline Species & Mean length $(\mu \mathrm{m})$ & Mean width $(\mu \mathrm{m})$ & $n$ & Longitudinal (somatic) kineties & Shape of methanogens \\
\hline Trimyema finlayi ${ }^{1}$ & 34.2 & 22.1 & 97 & $34-45$ & Polymorphic \\
\hline 'Trimyema sp.'2 & $\mathrm{n} / \mathrm{a}$ & $\mathrm{n} / \mathrm{a}$ & $\mathrm{n} / \mathrm{a}$ & $37-40$ & Polymorphic \\
\hline Trimyema compressum ${ }^{3}$ & 39.05 & 22.3 & 20 & $50-60$ & Rod \\
\hline Trimyema compressum ${ }^{4}$ & 65.9 & 54.6 & 48 & $50-60$ & Rod \\
\hline Trimyema compressum ${ }^{5}$ & 40 & 25 & $\mathrm{n} / \mathrm{a}$ & $\mathrm{n} / \mathrm{a}$ & Rod \\
\hline
\end{tabular}

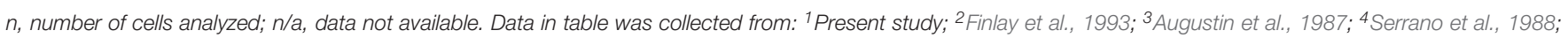

${ }^{5}$ Wagener and Pfennig, 1987.

errors. A comparable number of nucleotide differences (2 substitutions; 1616 compared bases) is also observed between the $18 \mathrm{~S}$ rRNA gene sequences from two isolates of T. compressum (accession numbers: AB285526.1 and Z29438.1). Some of the sequences included in the phylogenetic analysis shown in Figure 4 were obtained from environmental sequencing studies that have sampled a vast variety of geographical locations (Šlapeta et al., 2005; Zuendorf et al., 2006; Alexander et al., 2009; Takishita et al., 2010; Matsunaga et al., 2014; Pasulka et al., 2016). The ciliates from these studies are uncultured and 18S rRNA gene sequences provide the only evidence for their existence, which indicates that there is species-level diversity within the class Plagiopylea that remains uncharacterized.

Studies listed in Table 2 (Augustin et al., 1987; Wagener and Pfennig, 1987; Serrano et al., 1988), as well as a more recent study (Shinzato et al., 2007), describe T. compressum as having rod-shaped endosymbiotic methanogens, whereas fluorescence (Figures 2G-I) and TEM images (Figure 3) show that $T$. finlayi has irregularly-shaped endosymbiotic methanogens. Furthermore, the general morphology, cellular distribution and overall appearance of the endosymbionts from T. finlayi, as well as their associations with hydrogenosomes, appear to be very similar to previously published TEM images of 'Trimyema sp.' (Finlay et al., 1993). The 16S rRNA genes of the endosymbiotic methanogens in T. finlayi and 'Trimyema sp.' are $99.5 \%$ identical (2 substitutions; 443 compared bases) and phylogenetic analysis with other methanogen sequences (Figure 5) suggests that they are closely related to each other (bootstrap support $=100$, posterior probability $=0.98$ ) and belong to the genus Methanocorpusculum. In contrast, the endosymbiont of $T$. compressum is related to members of the genus Methanobrevibacter (Figure 5), which supports the findings of a previous study (Shinzato et al., 2007).

In addition to containing an endosymbiotic methanogen, T. compressum was previously shown to also contain a bacterial endosymbiont, closely related to the species Petrimonas sulfuriphila (Shinzato et al., 2007). We found no evidence however, from FISH experiments using a Bacteria-specific probe, to suggest that $T$. finlayi has a bacterial endosymbiont. 
Our findings provide robust morphological and molecular evidence to suggest that T. finlayi and 'Trimyema sp.' are two isolates of the same species, which from this point forward should be referred to as T. finlayi. We have also shown that this species is distinct from but closely related to T. compressum.

Previous studies have provided evidence that methanogenic endosymbionts of anaerobic ciliates do not co-speciate over the long-term with their hosts, suggesting that the endosymbionts of some anaerobic ciliates have occasionally been replaced by another species (Finlay et al., 1993; van Hoek et al., 2000b). Thus, closely-related hosts may have methanogen endosymbionts from different genera and vice versa (Embley and Finlay, 1994). Our results further support a lack of long-term co-speciation between host and symbionts in the Trimyema lineage - while the hosts T. compressum and T. finlayi (formerly 'Trimyema sp.') clearly belong to the same genus, the endosymbiotic methanogens of these two species are not closely related (Figure 5) (Shinzato and Kamagata, 2010). In the case of T. finlayi, however, there does appear to be stability of these associations in the evolutionary short-term (i.e., spatially and temporally isolated samples of the same species). Thus, T. finlayi (formerly 'Trimyema sp.') has now been isolated on two different occasions from distant geographical locations as part of separate studies, several years apart, and both isolates contain closely-related endosymbionts belonging to the genus Methanocorpusculum (Figure 5). T. finlayi was initially isolated from Priest Pot, a pond in Cumbria, northern England, United Kingdom (Finlay et al., 1993), and in the present study from a pond in East Stoke Fen, Dorset, southern England, United Kingdom. These two sites are separated by over $400 \mathrm{~km}$ and were sampled approximately 22 years apart. The finding that at least some anaerobic ciliates retain their endosymbiotic methanogens over the evolutionary short-term indicates that the symbiotic consortium is not entirely transient.

The observed Methanocorpusculum endosymbionts in T. finlayi are polymorphic (Finlay et al., 1993), and differed from the typical coccoid morphology of some of their closest known free-living relatives (Anderson et al., 2009). Some of the endosymbiont cells formed close associations with the ciliates hydrogenosomes, which is likely to be an adaptation to their endosymbiotic lifestyle, allowing them to uptake $\mathrm{H}_{2}$ with increased efficiency (Finlay et al., 1993). Similar observations have been made in the ciliate Metopus contortus, which also has polymorphic endosymbionts of the genus Methanocorpusculum, and also seem to undergo a morphological transformation (Embley et al., 1992a), suggesting that species of the genus Methanocorpusculum might share homologous adaptations that facilitate their endosymbiotic lifestyle.

The endosymbionts of $T$. finlayi appear to transform their morphology, presumably to form closer associations with hydrogenosomes, which suggests that these two organisms have evolved a relatively stable association. In contrast, although the endosymbionts of $T$. compressum can also be closely associated with hydrogenosomes (Shinzato et al., 2007), they typically appear rod-shaped and therefore resemble other free-living methanogen species of the same genus (Methanobrevibacter) (Wagener and Pfennig, 1987; Goosen et al., 1990). There are also reported cases where methanogenic endosymbionts were lost from T. compressum in laboratory cultures (Wagener and Pfennig, 1987; Wagener et al., 1990; Holler and Pfennig, 1991). In some of these cases the ciliates re-incorporated the endosymbionts when they were co-incubated with a pregrown methanogen culture (Wagener et al., 1990). These observations suggest that the endosymbiont of $T$. compressum may be less adapted to an endosymbiotic lifestyle, and provides evidence that the association between these species is less evolutionarily stable in comparison to the corresponding symbiosis in T. finlayi. Alternatively, the capacity to lose and subsequently re-establish endosymbionts within its cells could be a mechanism used by $T$. compressum to adapt to a changing environment.

Additional sampling, together with reliable in situ identification, of endosymbiotic methanogens living within other congeneric ciliate species, would provide further insight into the extent, or lack of, co-speciation between host and endosymbiont. Sequencing the genomes of the methanogenic endosymbionts from both $T$. finlayi and $T$. compressum, and comparing them with the genomes of their close free-living relatives, could also provide molecular insights into the relative stability of these associations, by identifying general or species-specific patterns of gene loss or gain that have allowed certain methanogens to become endosymbionts.

\section{AUTHOR CONTRIBUTIONS}

WL carried out field work, molecular lab work, microscopy and bioinformatic data analysis, and drafted the manuscript. KS carried out molecular lab work, TME coordinated and helped to design aspects of the study, and GE conceived the study, identified the species and carried out field and lab work.

\section{FUNDING}

WL was supported by a European Research Council Advanced Investigator Grant to TME (ERC-2010-AdG-268701). GE acknowledges financial support from the Alice Ellen Cooper Dean Trust (UK).

\section{ACKNOWLEDGMENTS}

We would like to thank the following people: A. Laude (Bioimaging Unit, Newcastle University, United Kingdom) for providing assistance with confocal imaging; E. Kozhevnikova (Newcastle University, United Kingdom) for help with various aspects of lab work that contributed to this research; B. Haenni and B. Zuber (University of Bern, Switzerland) for their electron microscopy work and expertise. 


\section{REFERENCES}

Alexander, E., Stock, A., Breiner, H. W., Behnke, A., Bunge, J., Yakimov, M. M., et al. (2009). Microbial eukaryotes in the hypersaline anoxic L'Atalante deep-sea basin. Environ. Microbiol. 11, 360-381. doi: 10.1111/j.1462-2920.2008.01777.x

Anderson, I. J., Sieprawska-Lupa, M., Goltsman, E., Lapidus, A., Copeland, A., Del Rio, T. G., et al. (2009). Complete genome sequence of Methanocorpusculum labreanum type strain Z. Stand. Genomic Sci. 1, 197-203. doi: 10.4056/sigs. 35575

Augustin, H., Foissner, W., and Adam, H. (1987). Revision of the genera Acoineria, Trimyema and Trochiliopsis (Protozoa, Ciliophora). Bull. Br. Mus. Nat. Hist. 52, 197-224.

Baumgartner, M., Stetter, K. O., and Foissner, W. (2002). Morphological, small subunit rRNA, and physiological characterization of Trimyema minutum (Kahl, 1931), an anaerobic ciliate from submarine hydrothermal vents growing from $28^{\circ} \mathrm{C}$ to $52^{\circ} \mathrm{C}$. J. Eukaryot. Microbiol. 49, 227-238. doi: 10.1111/j.1550-7408. 2002.tb00527.x

Broers, C. A., Stumm, C. K., Vogels, G. D., and Brugerolle, G. (1990). Psalteriomonas lanterna gen. nov., sp. nov., a free-living amoeboflagellate isolated from freshwater anaerobic sediments. Eur. J. Protistol. 25, 369-380. doi: 10.1016/S0932-4739(11)80130-6

Castresana, J. (2000). Selection of conserved blocks from multiple alignments for their use in phylogenetic analysis. Mol. Biol. Evol. 17, 540-552. doi: 10.1093/ oxfordjournals.molbev.a026334

Cho, B. C., Park, J. S., Xu, K., and Choi, J. K. (2008). Morphology and molecular phylogeny of Trimyema koreanum n. sp., a ciliate from the hypersaline water of a solar saltern. J. Eukaryot. Microbiol. 55, 417-426. doi: 10.1111/j.1550-7408. 2008.00340.x

Daims, H., Stoecker, K., and Wagner, M. (2005). "Fluorescence in situ hybridization for the detection of prokaryotes," in Molecular Microbial Ecology, eds A. M. Osborn and C. J. Smith (New York, NY: Taylor \& Francis Group), 213-239.

Darriba, D., Taboada, G. L., Doallo, R., and Posada, D. (2012). jModelTest 2: more models, new heuristics and parallel computing. Nat. Methods 9, 772-772. doi: $10.1038 /$ nmeth. 2109

Doddema, H., and Vogels, G. (1978). Improved identification of methanogenic bacteria by fluorescence microscopy. Appl. Environ. Microbiol. 36, 752-754.

Edgar, R. C. (2004). MUSCLE: multiple sequence alignment with high accuracy and high throughput. Nucleic Acids Res. 32, 1792-1797. doi: 10.1093/nar/gkh340

Embley, T. M. (2006). Multiple secondary origins of the anaerobic lifestyle in eukaryotes. Philos. Trans. R. Soc. Lond. B Biol. Sci. 361, 1055-1067. doi: 10.1098/ rstb.2006.1844

Embley, T. M., and Finlay, B. J. (1993). Systematic and morphological diversity of endosymbiotic methanogens in anaerobic ciliates. Antonie Van Leeuwenhoek 64, 261-271. doi: 10.1007/BF00873086

Embley, T. M., and Finlay, B. J. (1994). The use of small subunit rRNA sequences to unravel the relationships between anaerobic ciliates and their methanogen endosymbionts. Microbiology 140, 225-235. doi: 10.1099/13500872-140-2-225

Embley, T. M., Finlay, B. J., and Brown, S. (1992a). RNA sequence analysis shows that the symbionts in the ciliate Metopus contortus are polymorphs of a single methanogen species. FEMS Microbiol. Lett. 97, 57-61.

Embley, T. M., Finlay, B. J., Dyal, P. L., Hirt, R. P., Wilkinson, M., and Williams, A. G. (1995). Multiple origins of anaerobic ciliates with hydrogenosomes within the radiation of aerobic ciliates. Proc. Biol. Sci. 262, 87-93. doi: 10.1098/rspb. 1995.0180

Embley, T. M., Finlay, B. J., Thomas, R. H., and Dyal, P. L. (1992b). The use of rRNA sequences and fluorescent probes to investigate the phylogenetic positions of the anaerobic ciliate Metopus palaeformis and its archaeobacterial endosymbiont. J. Gen. Microbiol. 138, 1479-1487.

Embley, T. M., van der Giezen, M., Horner, D., Dyal, P., Bell, S., and Foster, P. (2003). Hydrogenosomes, mitochondria and early eukaryotic evolution. IUBMB Life 55, 387-395. doi: 10.1080/15216540310001592834

Esteban, G. F., and Finlay, B. J. (2004). Marine ciliates (Protozoa) in central Spain. Ophelia 58, 12-22. doi: 10.1080/00785236.2004.10410209

Fenchel, T., and Finlay, B. J. (1991). Synchronous division of an endosymbiotic methanogenic bacterium in the anaerobic ciliate Plagiopyla frontata Kahl. J. Protozool. 38, 22-28. doi: 10.1111/j.1550-7408.1991.tb04790.x

Fenchel, T., and Finlay, B. J. (1995). Ecology and Evolution in Anoxic Worlds. Oxford: Oxford University Press.
Fernández-Galiano, D. (1994). The ammoniacal silver carbonate method as a general procedure in the study of protozoa from sewage (and other) waters. Water Res. 28, 495-496. doi: 10.1016/0043-1354(94)90288-7

Finlay, B. J., Embley, T. M., and Fenchel, T. (1993). A new polymorphic methanogen, closely related to Methanocorpusculum parvum, living in stable symbiosis within the anaerobic ciliate Trimyema sp. J. Gen. Microbiol. 139, 371-378. doi: 10.1099/00221287-139-2-371

Finlay, B. J., Esteban, G., Clarke, K. J., Williams, A. G., Embley, T. M., and Hirt, R. P. (1994). Some rumen ciliates have endosymbiotic methanogens. FEMS Microbiol. Lett. 117, 157-161. doi: 10.1111/j.1574-6968.1994.tb06758.x

Finlay, B. J., and Fenchel, T. (1992). An anaerobic ciliate as a natural chemostat for the growth of endosymbiotic methanogens. Eur. J. Protistol. 28, 127-137. doi: 10.1016/S0932-4739(11)80041-6

Gantner, S., Andersson, A. F., Alonso-Sáez, L., and Bertilsson, S. (2011). Novel primers for $16 \mathrm{~S}$ rRNA-based archaeal community analyses in environmental samples. J. Microbiol. Methods 84, 12-18. doi: 10.1016/j.mimet.2010.10.001

Goosen, N. K., Wagener, S., and Stumm, C. K. (1990). A comparison of two strains of the anaerobic ciliate Trimyema compressum. Arch. Microbiol. 153, 187-192. doi: $10.1007 / \mathrm{BF} 00247819$

Holler, S., and Pfennig, N. (1991). Fermentation products of the anaerobic ciliate Trimyema compressum in monoxenic cultures. Arch. Microbiol. 156, 327-334. doi: 10.1007/BF00263006

López-García, P., Rodríguez-Valera, F., Pedrós-Alió, C., and Moreira, D. (2001). Unexpected diversity of small eukaryotes in deep-sea Antarctic plankton. Nature 409, 603-607. doi: 10.1038/35054537

Lynn, D. (2008). The Ciliated Protozoa: Characterization, Classification, and Guide to the Literature. Berlin: Springer Science \& Business Media.

Matsunaga, K., Kubota, K., and Harada, H. (2014). Molecular diversity of eukaryotes in municipal wastewater treatment processes as revealed by $18 \mathrm{~S}$ rRNA gene analysis. Microbes Environ. 29, 401-407. doi: 10.1264/jsme2. ME14112

Pasulka, A. L., Levin, L. A., Steele, J. A., Case, D. H., Landry, M. R., and Orphan, V. J. (2016). Microbial eukaryotic distributions and diversity patterns in a deepsea methane seep ecosystem. Environ. Microbiol. 18, 3022-3043. doi: 10.1111/ 1462-2920.13185

Ronquist, F., and Huelsenbeck, J. P. (2003). MrBayes 3: bayesian phylogenetic inference under mixed models. Bioinformatics 19, 1572-1574. doi: 10.1093/ bioinformatics/btg180

Schindelin, J., Arganda-Carreras, I., Frise, E., Kaynig, V., Longair, M., Pietzsch, T., et al. (2012). Fiji: an open-source platform for biological-image analysis. Nat. Methods 9, 676-682. doi: 10.1038/nmeth.2019

Serrano, S., Martín-González, A., and Fernández-Galiano, D. (1988). Trimyema compressum Lackey, 1925: morphology, morphogenesis and systematic implications. J. Protozool. 35, 315-320. doi: 10.1111/j.1550-7408.1988.tb 04349.x

Shinzato, N., and Kamagata, Y. (2010). "The methanogenic and eubacterial endosymbionts of Trimyema," in (Endo)symbiotic Methanogenic Archaea. Microbiology Monographs, Vol. 19, ed. J. Hackstein (Berlin: Springer), 35-53. doi: 10.1007/978-3-642-13615-3_4

Shinzato, N., Watanabe, I., Meng, X.-Y., Sekiguchi, Y., Tamaki, H., Matsui, T., et al. (2007). Phylogenetic analysis and fluorescence in situ hybridization detection of archaeal and bacterial endosymbionts in the anaerobic ciliate Trimyema compressum. Microb. Ecol. 54, 627-636. doi: 10.1007/s00248-007-9218-1

Šlapeta, J., Moreira, D., and López-García, P. (2005). The extent of protist diversity: insights from molecular ecology of freshwater eukaryotes. Proc. Biol. Sci. 272, 2073-2081. doi: 10.1098/rspb.2005.3195

Stahl, A. D., and Amann, R. (1991). "Development and application of nucleic acid probes," in Nucleic Acid Techniques in Bacterial Systematics, eds M. Goodfellow and E. Stackebrandt (New York, NY: John Wiley \& Sons), 205-248.

Stamatakis, A. (2014). RAxML version 8: a tool for phylogenetic analysis and post-analysis of large phylogenies. Bioinformatics 30, 1312-1313. doi: 10.1093/ bioinformatics/btu033

Takishita, K., Kakizoe, N., Yoshida, T., and Maruyama, T. (2010). Molecular evidence that phylogenetically diverged ciliates are active in microbial mats of deep-sea cold-seep sediment. J. Eukaryot. Microbiol. 57, 76-86. doi: 10.1111/j. 1550-7408.2009.00457.x

Tschanz, S. A., Makanya, A. N., Haenni, B., and Burri, P. H. (2003). Effects of neonatal high-dose short-term glucocorticoid treatment on the lung: a 
morphologic and morphometric study in the rat. Pediatr. Res. 53, 72-80. doi: 10.1203/00006450-200301000-00014

van Bruggen, J. J., Stumm, C. K., and Vogels, G. D. (1983). Symbiosis of methanogenic bacteria and sapropelic protozoa. Arch. Microbiol. 136, 89-95. doi: $10.1007 /$ BF00404779

van Bruggen, J. J., Stumm, C. K., Zwart, K. B., and Vogels, G. D. (1985). Endosymbiotic methanogenic bacteria of the sapropelic amoeba Mastigella. FEMS Microbiol. Lett. 31, 187-192. doi: 10.1111/j.1574-6968.1985.tb01147.x

van Hoek, A. H., Akhmanova, A. S., Huynen, M. A., and Hackstein, J. H. (2000a). A mitochondrial ancestry of the hydrogenosomes of Nyctotherus ovalis. Mol. Biol. Evol. 17, 202-206. doi: 10.1093/oxfordjournals.molbev.a026234

van Hoek, A. H., van Alen, T. A., Sprakel, V. S., Leunissen, J. A., Brigge, T., Vogels, G. D., et al. (2000b). Multiple acquisition of methanogenic archaeal symbionts by anaerobic ciliates. Mol. Biol. Evol. 17, 251-258.

Wagener, S., Bardele, C., and Pfennig, N. (1990). Functional integration of Methanobacterium formicicum into the anaerobic ciliate Trimyema compressum. Arch. Microbiol. 153, 496-501. doi: 10.1007/BF00248433

Wagener, S., and Pfennig, N. (1987). Monoxenic culture of the anaerobic ciliate Trimyema compressum Lackey. Arch. Microbiol. 149, 4-11. doi: 10.1007/ BF00423128
Zuendorf, A., Bunge, J., Behnke, A., Barger, K. J. A., and Stoeck, T. (2006). Diversity estimates of microeukaryotes below the chemocline of the anoxic Mariager Fjord, Denmark. FEMS Microbiol. Ecol. 58, 476-491. doi: 10.1111/j.1574-6941. 2006.00171.x

Zwart, K. B., Goosen, N. K., Van Schijnde, M. W., Broers, C. A., Stumm, C. K., and Vogels, G. D. (1988). Cytochemical localization of hydrogenase activity in the anaerobic protozoa Trichomonas vaginalis, Plagiopyla nasuta and Trimyema compressum. Microbiology 134, 2165-2170. doi: 10.1099/00221287-134-8-2165

Conflict of Interest Statement: The authors declare that the research was conducted in the absence of any commercial or financial relationships that could be construed as a potential conflict of interest.

Copyright (c) 2018 Lewis, Sendra, Embley and Esteban. This is an open-access article distributed under the terms of the Creative Commons Attribution License (CC BY). The use, distribution or reproduction in other forums is permitted, provided the original author(s) and the copyright owner are credited and that the original publication in this journal is cited, in accordance with accepted academic practice. No use, distribution or reproduction is permitted which does not comply with these terms. 